\title{
A Dual-Mode Nested Rectifier for Ambient Wireless Powering in CMOS Technology
}

\author{
Abdullah S. Almansouri, Student Member, IEEE, Jürgen Kosel, Senior Member, IEEE and Khaled N. \\ Salama, Senior Member, IEEE
}

\begin{abstract}
This paper proposes a dual-mode nested RF rectifier for ambient wireless powering. The proposed architecture utilizes a dual-mode nested feedback circuit to enhance the conductivity of the rectifier at low-power while reducing the reverse leakage current at high-power by generating supply voltages at the gates of the PMOS rectifying transistors. The proposed rectifier is fabricated in $65 \mathrm{~nm}$ CMOS technology and occupies an area of $6480 \mathrm{\mu m}^{2}$. The measurement results show a peak power conversion efficiency of $86 \%, 10.1 \mathrm{~dB}$ dynamic range, and $-19.2 \mathrm{dBm} 1-\mathrm{V}$ sensitivity when operating with a $100 \mathrm{k} \Omega$ load at the industrial, scientific and medical band $433 \mathrm{MHz}$. Moreover, the enhanced low-power performance is achieved by reducing the effective threshold voltage of the rectifier by about $37 \%$, compared to a lowthreshold transistor in $65 \mathrm{~nm}$ technology. This reduction in the threshold voltage allows the rectifier to operate with efficiency exceeding $10 \%$ for input power $\geq-40 \mathrm{dBm}$.
\end{abstract}

Index Terms - Ambient wireless powering, dual-mode, energy harvesting, nested rectifier, RF-DC converter, self-bias, rectifier, wireless power transfer, wireless powering.

\section{INTRODUCTION}

$\mathrm{O}$ VER THE past two decades, we witnessed the revolution of wireless sensor network (WSN) and the associated fields, including, Internet of Things (IoT), wearable and implantable biomonitoring devices, machine to machine communication and cyber-physical systems [1]-[3]. One of the visions of WSN is to autonomously monitor the environmental parameters such as temperature, moisture, and dissolved oxygen [4], [5]. The WSN market was valued at USD 29.06 Billion at 2016, and it is expected to reach USD 93.86 Billion by 2023 [6]. Powering such a considerable number of WSN devices using conventional methods (i.e., using wireline connections or batteries) can be very challenging or even impossible [2], [7]. Thus, wireless power transfer (WPT) has been heavily investigated as a practical approach for powering battery-free WSN [7]-[16]. With the recent enhancement in WPT techniques and the efficiency of the integrated circuits, the vision of having a truly WSN is becoming a reality [17][28].

WPT can be classified into near-field WPT that delivers tens of watts for a short distance (i.e., less than one meter); and microwave WPT that delivers RF power over large distances (i.e., as long as within the range of an RF transmitter).

A. S. Almansouri, J. Kosel and K. N. Salama are with King Abdullah University of Science and Technology (KAUST), Thuwal 23955-6900, Saudi Arabia (e- mail: abdullah.almansouri@kaust.edu.sa;
Depending on the distance between the RF transmitter and the microwave WPT receiver, the power level at the receiver can vary from milliwatts to microwatts [15]. The microwave WPT utilizes a wide range of frequencies including high frequency (HF) band and ultra-high frequency (UHF) band [29]. One of which is the industrial, scientific and medical $433 \mathrm{MHz}$ band that is used for license-free communication, automotive applications [30] and underwater wireless-sensor networks [31].

Fig. 1 shows a schematic of a wireless power receiver (WPR) for wireless powering. It consists of an antenna, a matching network, a radio frequency (RF) rectifier, and a storage element. The RF rectifier is a critical component as it is responsible for converting the limited RF power into a dc voltage. In fact, it determines the sensitivity of the WPR defined as the required input power to generate a certain amount of dc output voltage; the dynamic range (DR) which is the range of input power where the WPR operates efficiently; and a significant portion of the overall power conversion efficiency (PCE) [32]. The efficient operation range of the WPR is determined by 1) the threshold voltage of the rectifier and 2) the reverse leakage current (IREV) in which some amount of the harvested energy leaks back the $R F$ input. Ideally, to extended the efficient operating range of the WPR there is a need to minimize both of the threshold voltage and the IREV.

Many works have been proposed to improve the overall performance of the RF rectifier. Traditionally, rectifiers have been realized using diode-based architectures, such as Dickson [33], half-wave and full-wave rectifiers [34]. In CMOS technology, diodes are realized using diode-connected

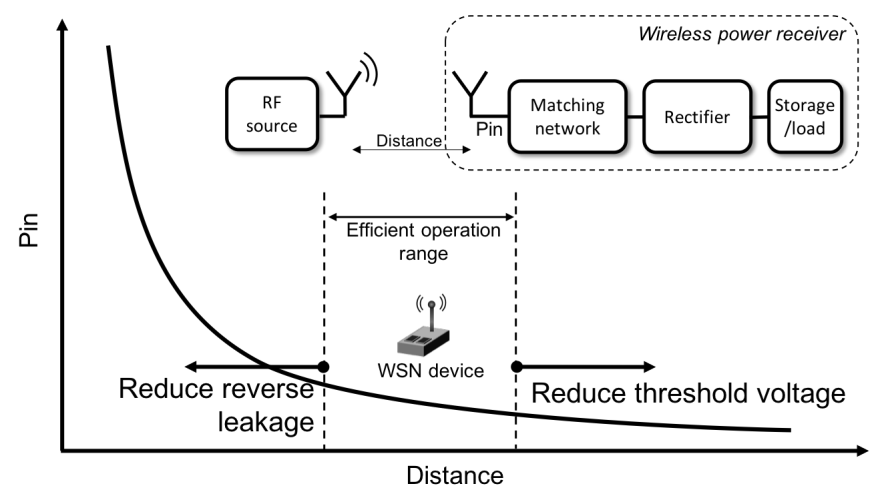

Fig. 1. Efficient operation region of the wireless power receiver. Inset is a block diagram of a wireless power receiver.

jurgen.kosel@kasut.edu.sa; khaled.salama@kaust.edu.sa). A. S. Almansouri is also with the University of Jeddah, Jeddah, Saudi Arabia 


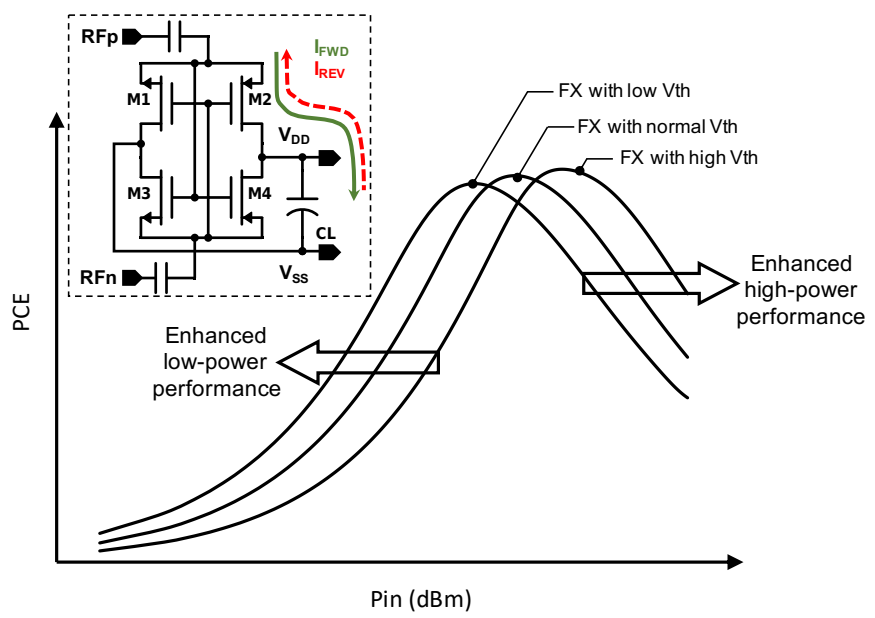

Fig. 2. Impact of changing the threshold voltage of the rectifying transistors (M1-4) on the performance of the FX rectifier. Inset is schematic of the FX architecture

transistors where the gate and the drain of a transistor are connected together. However, these rectifiers suffer from poor low-power performance and poor PCE due to the high dropout voltage across the diodes [35]. Non-CMOS technologies such as Schottky diodes can enhance the low-power performance of the diode-based rectifiers [3], [36]. However, it is seldom offered in conventional CMOS processes; hence, it cannot be used in lowcost applications where high integration level is needed [3], [35]. Fully cross-coupled (FX) architecture came to solve this problem by applying the input power differentially over a crosscouple bridge, as shown in Fig. 2 inset [37]. Such a configuration offers relatively good low-power performance and peak PCE in standard CMOS technology. However, the FX architecture suffers from poor high-power performance and poor DR due to the presence of the IREV which leaks part of the harvested energy back to the RF input [38], [39]. Many self-biased architectures were proposed to reduce the IREV by lowering the driving voltage of the rectifying transistors at high input power [35], [38], [40]. As a consequence, the self-biased based architectures extended the efficient operating range of the rectifier by offering a wider DR.

Depending on the input power, the rectifiers might not be able to generate sufficient dc voltage for the proper operation of the WPR [41]. For such cases, some of these architectures (i.e., Dickson, FX, self-biased) are arranged in a way to act as a voltage multiplier [33], [37], [41]. For example, voltage multiplier using the FX architecture is achieved by several FX stages connected in series for the dc path where the $V_{D D}$ terminal of the first stage is connected to the $V_{S S}$ of the next stage, and in parallel for the RF input [37]. Still, the minimum operating input power of a rectifier is limited by the threshold voltage of the transistors. One way to solve this problem is by using a lowthreshold transistor for the FX architecture. However, by using low threshold transistors the IREV increases, hence, worsening the high-power performance, as shown in Fig. 2. Lowering the threshold voltage of the FX architecture shifts the efficient operating region toward the lower input power range by sacrificing the high-power PCE performance. To this end, Schottky diode-based architectures perform better at very low input power due to their low threshold voltage [3], [18], [41], and there is a need to reduce the effective threshold voltage of the CMOS-based architectures without sacrificing the PCE at high input power.

This work proposes a highly efficient dual-mode nested RFto-DC converter for ambient wireless powering. The proposed rectifier is based on the FX architecture and extends the concept of self-bias to enhance both the low- and high- power performance. The proposed design lowers the effective threshold voltage of the rectifier, offers higher PCE at low and high input power, a wider DR and higher $1-\mathrm{V}$ sensitivity, compared to both the FX and the self-biased architectures. This paper is organized as follows: Section II introduces the dualmode nested feedback circuit and explains the working methodology, Section III shows the measurement results, and Section IV presents conclusions.

\section{Proposed Design}

\section{A. Concept}

Conceptually, to improve the performance of the FX rectifier at low input power, there is a need to enhance the conductivity of the rectifying transistors (i.e., by increasing the overdrive voltage of the rectifying transistors). On the other hand, to enhance the performance of the FX rectifier at high input power (i.e., when the instantaneous RF voltage at the source of the rectifying transistors $\leq V D D-\mathrm{Vth}$ ), there is a need to reduce the $\mathrm{I}_{\mathrm{REV}}$ (i.e., by decreasing the overdrive voltage of the rectifying transistors). To achieve such performance, there is a need for a dual-mode (DM) feedback circuit that dynamically changes the $\mathrm{dc}$ operating points of the rectifying transistors, hence, changing the effective threshold voltage of the rectifier. Note that, the effiective threshold voltage is defined as the minimum voltage required to turn the rectifier $\mathrm{ON}$. The DM functionality is achieved by generating a $V_{S S}$ or $V_{D D}$ biasing voltage at the gates of the rectifying transistors $(\mathrm{M} 2,4)$ depending on the input power, as shown in Fig. 3. For example, to enhance the performance of the PMOS transistors $(\mathrm{M} 2,4)$ at low input power, $V_{S S}$ dc voltage is applied at the gates; and to enhance the performance of the rectifier at high input power, $V_{d d}$ dc voltage is applied at the gates.

The real challenge in realizing the DM concept is the lack of sufficient supply voltage at low input power for powering the feedback circuit. As a consequence, the use of inverters, switches or comparators is not appropriate for enhancing the

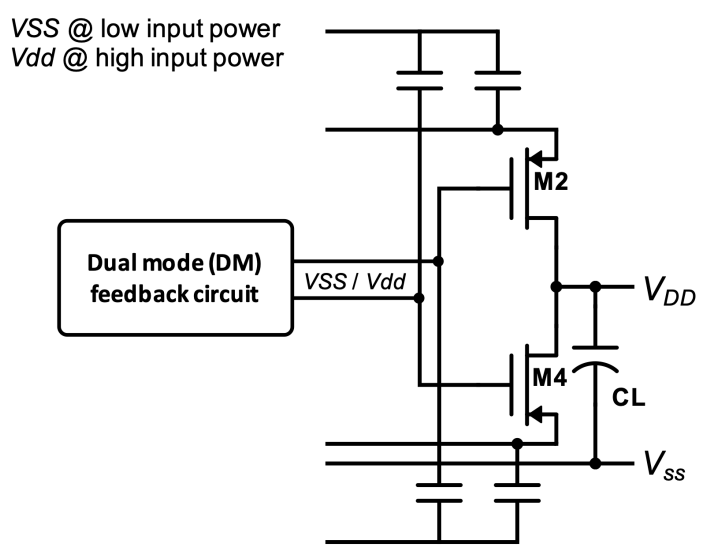

Fig. 3. Block diagram of the proposed dual-mode feedback circuit 
low-power performance of an RF rectifier. Therefore, the DM feedback circuit should be independent and self-sustained by harvesting its own energy. At the same time, the DM feedback circuit should consume minimum energy across the full range of input power to conserve the overall PCE. To overcome these challenges, a novel nesting scheme is proposed and discussed in depth in section IIB. Basically, the nested DM rectifier is achieved by utilizing two rectifiers acting as the DM feedback circuit and connected to the gates of M2 and M4. For such a configuration the output dc voltages of the nested rectifiers are used to bias the rectifying transistors of the main rectifier.

\section{B. Architecture}

Fig. 4(a) shows a schematic of the proposed DM rectifier for the enhanced performance. It consists of four low-threshold $\left(\mathrm{V}_{\text {th_L }}\right.$ ) rectifying transistors (M1-4), a DM feedback circuit that encloses two identical nested rectifying circuits connected to the gates of M2,4, six diode-connected transistors (D1-6), and eight coupling capacitors.

The DM feedback circuit consists of a weak-conduction lowthreshold voltage transistor $(\mathrm{M} 5,6)$ connected in parallel with a high-threshold $\left(\mathrm{V}_{\text {th_H }}\right)$ diode $(\mathrm{D} 7,8)$. Note that, the weakconduction of M5,6 and D7,8 is achieved using transistors with a small width to length ratio (W/L) to reduce the current flow. In such a configuration, M5,6 produces a $V_{S S}$ de signal at the gate of M2,4 at low input power, while D7,8 produces a $V_{d d}$ dc voltage signal at high input power. The DM feedback circuit by itself acts as a special type of a rectifier where the positive terminal (the cathode of D7,8) and the negative terminal (the drain of M5,6) are shorted together and connected the gates of M2,4. Note that, M5,6 is indeed inherited and equivalent to M1,3 in the FX design, and D7,8 is inherited and equivalent to the diode in the half-bridge rectifier. Since D7,8 is a highthreshold diode, its operation is limited to relatively high input power level (i.e., when the instantaneous RF voltage becomes

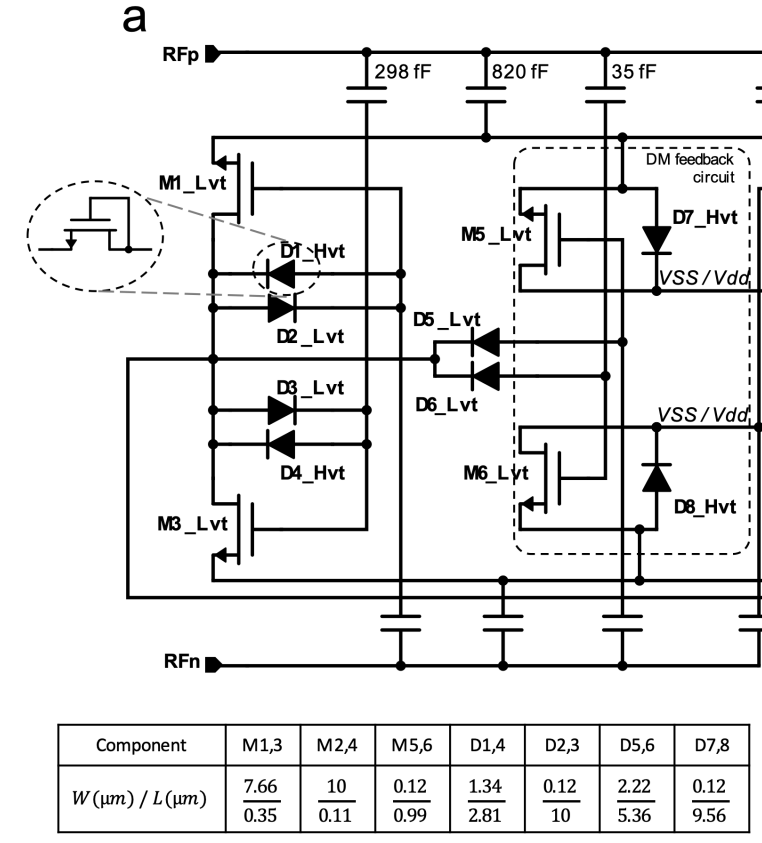

larger than $\mathrm{V}_{\text {th_ }}$ ). On the other hand, the low threshold voltage of M5,6 results in a pronounced operation at low input power. For such a configuration, the proposed DM feedback circuit is able to generate a $V_{S S}$ de voltage at low input power and a $V_{D D}$ voltage at high input power.

To reduce the short-circuit current between D7,8 and M5,6 at high input power (i.e., when both M5,6 and D7,8 are ON), D5,6 is connected to the gate of M5,6. D5,6 acts as a switch to reduce the conduction of M5, 6 by lowering the dc voltage at the gate of M5,6 at high power levels. In general, this concept is presented in [40]. In short, at low input power, the voltage-drop across D5,6 is relatively low (less than the threshold voltage of the diode); hence, the diode remains OFF and acts as an opencircuit. However, at relatively high input power, the voltagedrop across D5,6 exceeds the threshold voltage of the diodes. As a consequence, the diode is $\mathrm{ON}$ and drains current from the gate of M5,6, hence, lowering the dc voltage at M5,6's gate. Note that, D5,6 is a weak-conduction diode to reduce the leakage DC current from the DM feedback circuit to the load (i.e., $V_{S S}$ terminal). Similarly, the high-threshold diode D1,2 is connected to the gate of M1,3 to lower the dc voltage, hence, reducing $I_{\text {REV }}$ in M1,3 at high input power. On the other hand, D2, 4 enhances the IFWD of M1,3 by draining more current from the negative terminal of the load $\left(V_{S S}\right)$.

Fig. 4(b) shows the steady-state operating points of M2 (similarly M4). At low input power, M6 is active, and D8 is OFF. For such case, the driving voltage $\left(\mathrm{V}_{\mathrm{SG}}\right)$ of $\mathrm{M} 2$ is:

$$
V_{S G_{-} \text {low } \text { power_M2,4 }}=V_{R F}-V_{S S}
$$

where $V_{R F}$ is the instantaneous RF voltage, and $V_{S S}$ is the negative dc voltage supplied by the DM feedback circuit. Such high driving voltage is important at low input power where IREV is negligible and the need to enhance IFWD is critical. On the other hand, at high input power, D8 is active and M6 is OFF, as shown in Fig. 4(c). For such case, the driving voltage $\left(\mathrm{V}_{\mathrm{SG}}\right)$ of 
TABLE I.

DRIVING VOLTAGE FOR THE DIFFERENT SELF-BIASED BASED ARCHITECTURES AT LOW AND High INPUT POWER

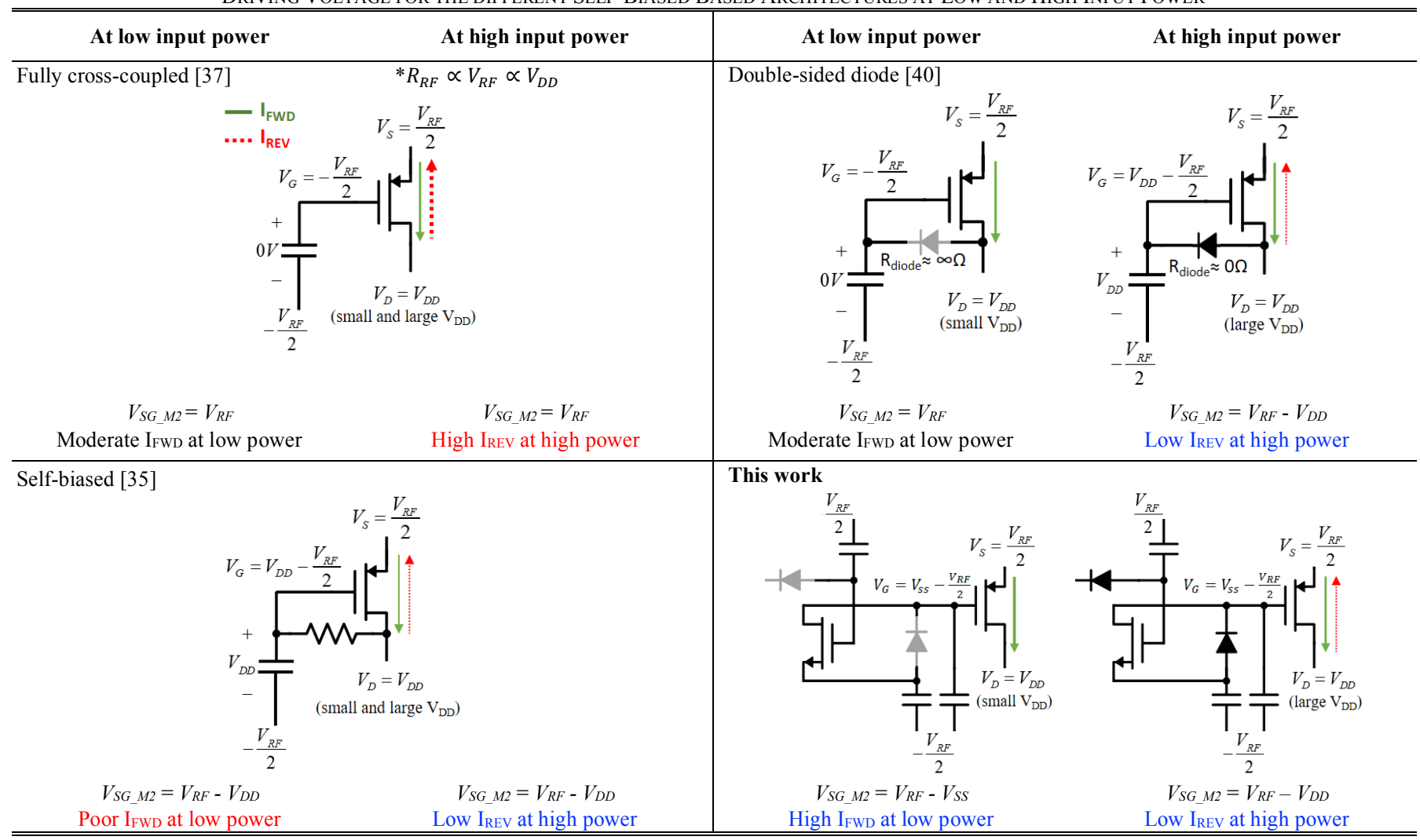

M2 is:

$V_{S G_{-} \text {high power_M2,4 }}=V_{R F}-V_{d d} \approx V_{R F}-V_{D D}$

where $V_{d d}$ is the positive de voltage supplied by the DM feedback circuit. Such small driving voltage is essential at high input power where reducing I IEV is critical. Table I compares the driving voltages of the different self-biased based architectures. As shown, the proposed DM feedback circuit is the only architecture with a high driving voltage at low input power allowing for high IFWD. It also maintains the enhanced performance at high input power levels by reducing IREv. Fig. 5 shows the simulation results of the output voltage and the normalized output voltage from the DM feedback circuit at both low- and high- input power. At low input power, the DM

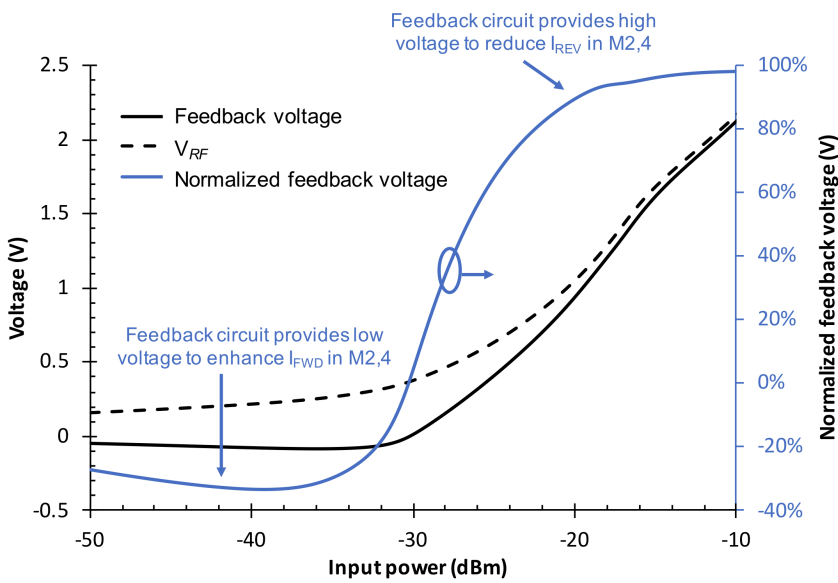

Fig. 5. Simulation results of the feedback voltage and the input RF voltage, and the feedback voltage normalized to the input RF voltage versus the input power for the proposed DM rectifier, operating at $433 \mathrm{MHz}$ with a $100 \mathrm{k} \Omega$ load. feedback circuit is capable of generating a low dc voltage to enhance the conductivity of M2,4. On the other hand, the DM feedback circuit is capable of generating a high dc voltage at high input power to reduce the reverse leakage current in M2,4.

Fig. 6 shows the performance for different types of rectifiers including the proposed DM rectifier, FX, half-wave and full wave rectifiers, simulated in $65 \mathrm{~nm}$ CMOS technology, 433 $\mathrm{MHz}$ frequency and with a $100 \mathrm{k} \Omega$ load. The simulation results indicate that the proposed DM rectifier has a significantly lower effective threshold voltage of about $170 \mathrm{mV}$, compared to the other architectures, as indicated in Fig. 6(a). Note that the reduction of the effective threshold voltage of the proposed DM rectifier below the actual threshold-voltage (i.e., about $270 \mathrm{mV}$ for a low threshold NMOS transistor in $65 \mathrm{~nm}$ technology, as anticipated from the performance of the half bridge rectifier) is due to the subthreshold conduction (I $\mathrm{I}_{\text {subthreshold }}$ ) of M5,6, which is described by:

$$
\begin{aligned}
I_{\text {subthreshold }} & =I_{d s 0} e^{\frac{V_{g s}-V_{t h}}{n v_{T}}}\left(1-e^{\frac{-V_{d s}}{v_{T}}}\right) \\
& =I_{d s 0} e^{\frac{V_{R F}-V_{t h}}{n v_{T}}}\left(1-e^{\frac{0.5 V_{R F}-V_{g, M 2,4}}{v_{T}}}\right)
\end{aligned}
$$

where $n$ and $I_{d s 0}$ are process dependent parameters, $v_{T}$ is the thermal voltage, $V_{g, M 2,4}$ is the dc voltage at the gate of M2,4, and $V_{t h}$ in the threshold voltage of M5,6 [42]. I $I_{\text {subthreshold }}$ results in the charging the equivalent capacitance at the gates of $\mathrm{M} 2,4$, hence, changing the dc operating points of M2,4 even when the RF voltage is below the threshold voltage. For such case, the dc voltage at the gates of M2,4 is equal to: 

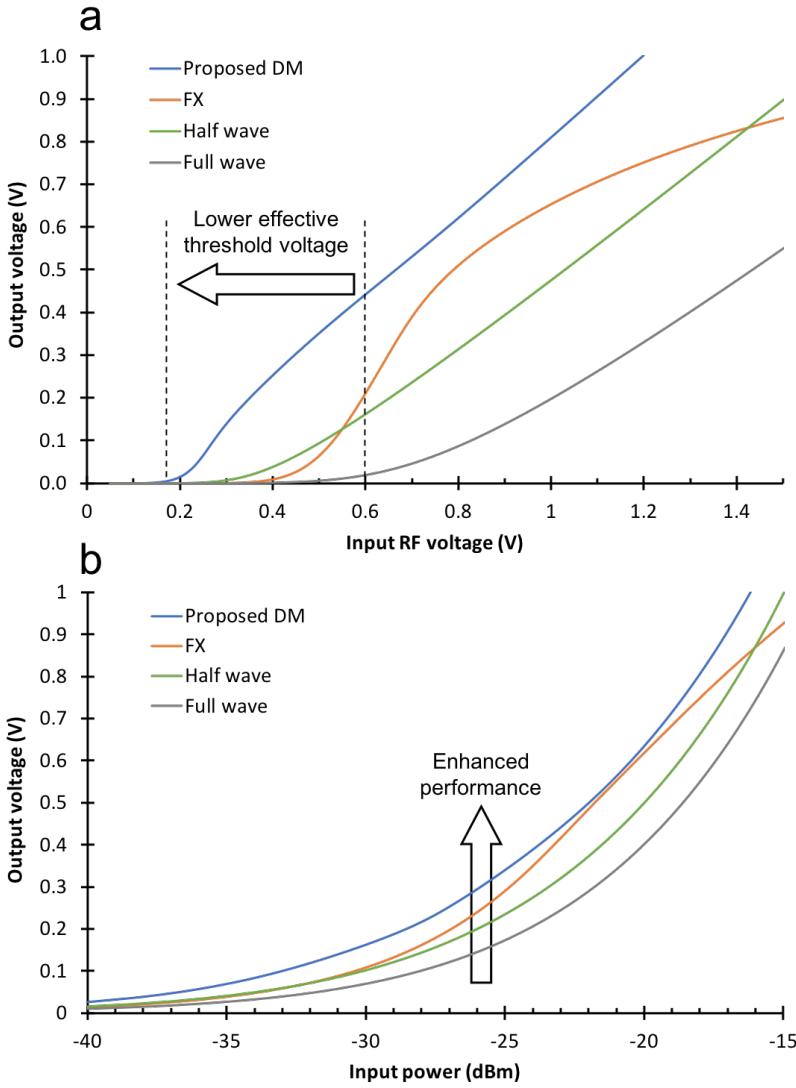

Fig. 6. Simulation results of (a) the output voltage versus the input voltage and (b) the output voltage versus the input power for different architectures, operating at $433 \mathrm{MHz}$ with a $100 \mathrm{k} \Omega$ load.

$$
V_{\text {sub }}=\frac{1}{C_{\text {total }}} \int I_{\text {subthreshold }} d t
$$

where $C_{\text {total }}$ is the total equivalent capacitance seen at the node connected the gate of $\mathrm{M} 2,4$, and approximated as:

$$
C_{\text {total }} \approx C_{g, M 2,4} / / C_{D 7,8} / / C_{\text {coupling }}
$$

where $C_{g, M 2,4}$ is the gate capacitance of $\mathrm{M} 2,4$, and $C_{D 7,8}$ is the equivalent capacitance looking at the cathode of D7,8. As a consequence, the effective threshold voltage of the rectifier is lowered by $V_{\text {sub }}$, and it equals:

$$
V_{t h \_ \text {eff }}=\left|V_{t h}\right|-\left|V_{d c_{-}, M 2,4}\right|=\left|V_{t h}\right|-\left|V_{s u b}\right|
$$

This reduction in the effective threshold voltage is reflected in the overall performance of the rectifier at low input power, as shown in Fig. 6(b) and Fig. 7(a). In fact, the dual-mode capability of the proposed architecture allows for consistently efficient performance across an extended range of the input power, resulting in higher output voltage and PCE at low- and high- input power levels, compared to the other architectures. Note, the PCE results for the DM rectifier include the losses in the feedback circuit and the leakage current. The simulation results in Fig. 7(a) indicates more than 100\% PCE enhancement compared to the FX when the input power is less than $-30 \mathrm{dBm}$. This enhanced performance of the DM rectifier is maintained
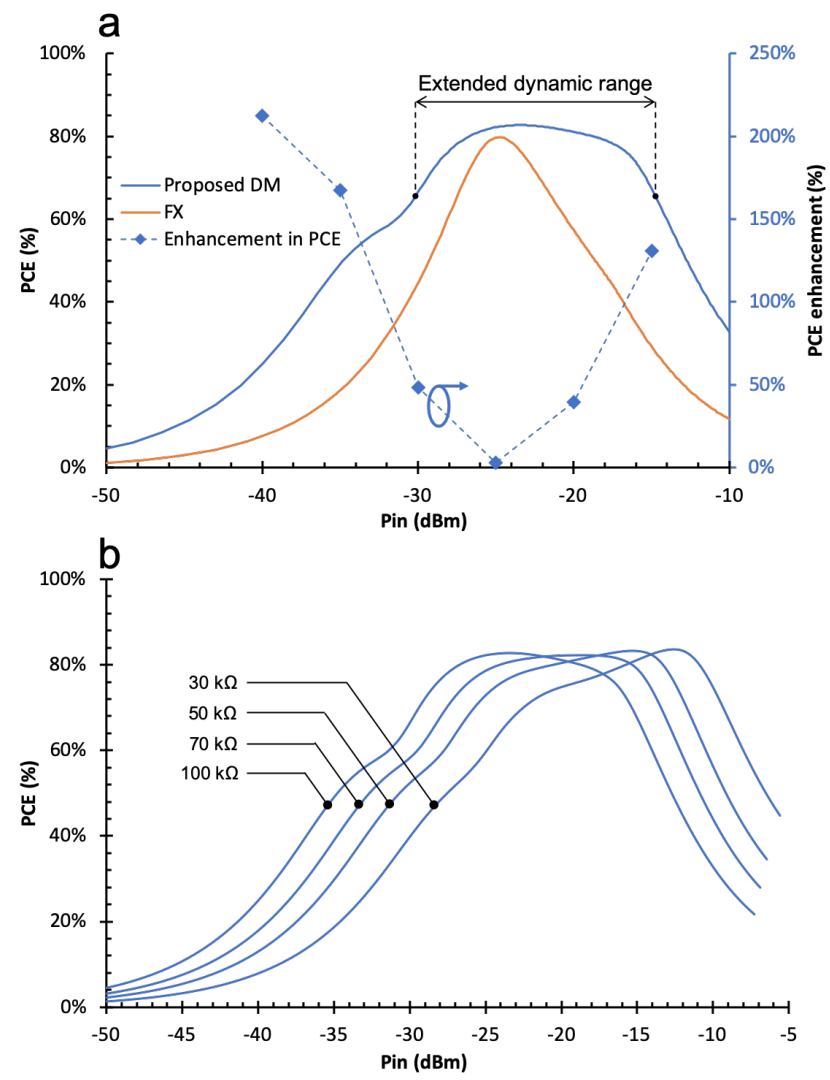

Fig. 7. Simulation results of (a) the PCE and the enhancement in the PCE versus the input power for the proposed DM and the FX rectifiers operating with a $100 \mathrm{k} \Omega$ load, and (b) the PCE of the proposed DM rectifier for different loads. Operating frequency is $433 \mathrm{MHz}$.

for different loads (i.e., $R_{\text {Load }}=30,50,70$ and $100 \mathrm{k} \Omega$ ), as shown in Fig. 7(b).

\section{MEASUREMENT RESUltS AND DISCUSSION}

The proposed DM rectifier is implemented in $65 \mathrm{~nm}$ standard CMOS technology. For a fair comparison, the FX architecture is also fabricated on the same die. Note that, the rectifying transistors (M1-4) for both architectures are low-threshold transistors. Also, the FX architecture was optimized separately for its optimum performance (FX sizing: M1,3 = $1480 \mu \mathrm{m} / 0.18$ $\mu \mathrm{m}$ and $\mathrm{M} 2,4=4 \mu \mathrm{m} / 0.1 \mu \mathrm{m})$. Fig. 8 shows a microphotograph of both architectures. The proposed DM rectifier occupies an area of $6.48 \times 10^{3} \mu \mathrm{m}^{2}$, compared to $0.43 \times 10^{3} \mu \mathrm{m}^{2}$. This increase in the area for the DM architecture is mostly due to the extra coupling capacitors introduced in the DM architectures.

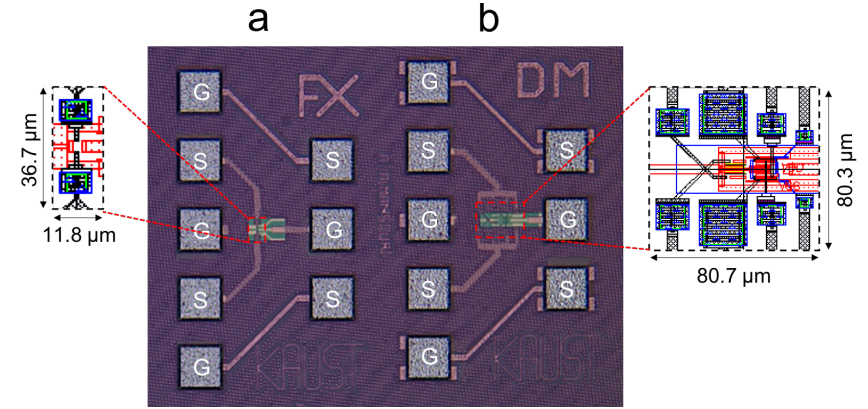

Fig. 8. Die microphotograph of (a) the FX and (b) the proposed DM architectures. Insets show pictures of the layouts. 
The measurement setup consists of a vector network analyzer (VNA) (Agilent N5225A), a digital multimeter (Keysight $34420 \mathrm{~A}$ ), and a $100 \mathrm{k} \Omega$ load. The test is achieved by RF probing the chip using a GSGSG differential probe with a reference plane set to the on-chip pads of the rectifier's input. After that, the RF power of the VNA is swept, and the corresponding Sparameters and the output voltage at the load are recorded. The instantaneous input power delivered to the rectifier is calculated by de-embedding the transmission and the reflection losses, as described by the following equation:

$$
P_{\text {in }}=P_{\text {source }}(d B m)-L_{\text {cable }}(d B)-10 \log \left|S_{11 \text { rect }}\right|^{2}
$$

where $P_{\text {source }}$ is the output RF power supplied by the VNA, $L_{\text {cable }}$ is the losses of the RF cable, and $S_{11 \text { rect }}$ is the measured S-parameters of the rectifier's input. Finally, the PCE of the rectifier is calculated as follow:

$$
\text { PCE }=\frac{P_{\text {out }}}{P_{\text {in }}}=\frac{v_{\text {out }}{ }^{2} / R_{\text {Load }}}{P_{\text {in }}}
$$

where $P_{\text {out }}$ is the output power delivered to the load, $v_{\text {out }}$ is the output voltage, and $R_{\text {Load }}$ is the $100 \mathrm{k} \Omega$ load [38], [35]. Note, the complete design of the WPT receivers (i.e., including the matching network and the antenna) using FX-based architectures are discussed in [43]-[45].

Fig. 9(a) shows the measured PCE for both the proposed DM and the FX architectures which is in good agreement with the simulation results. In general, the proposed design offers an
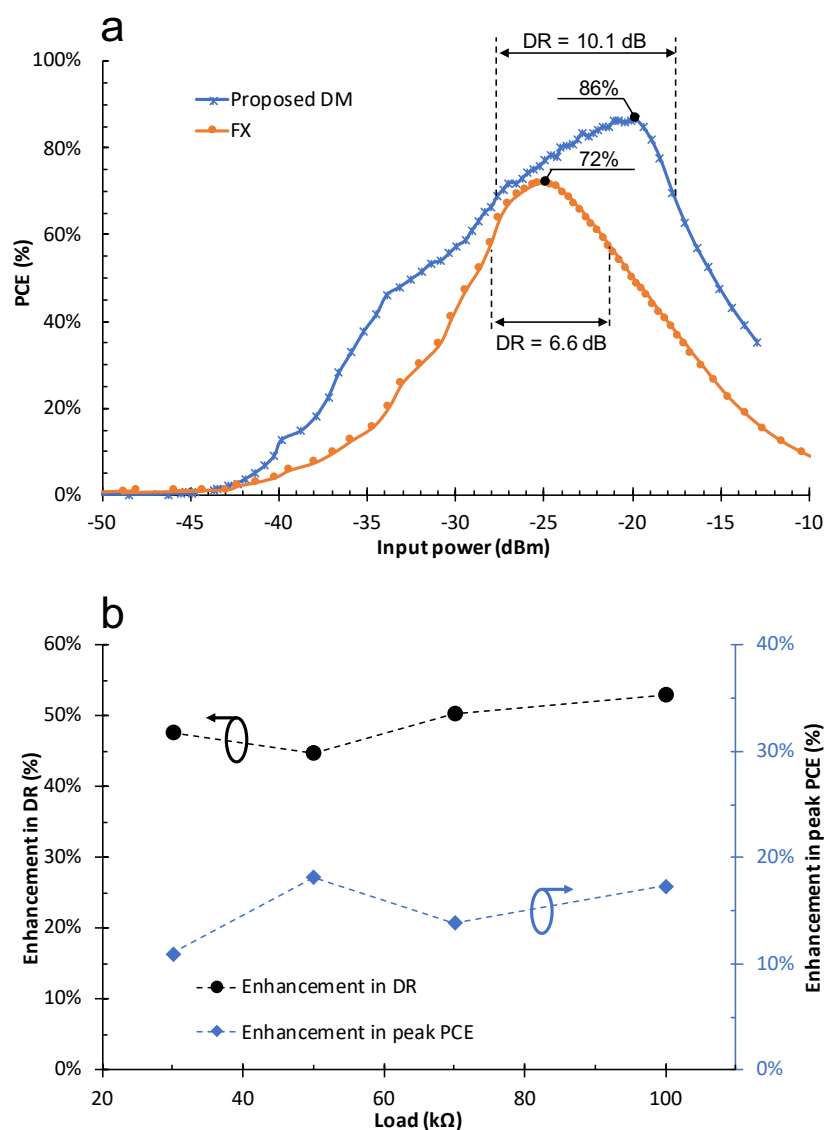

Fig. 9. (a) Measured PCE versus the input power with a $100 \mathrm{k} \Omega$ load, and (b) the measured enhancement in the dynamic range and the peak PCE for different loads. enhanced performance across the full range of the input power with a peak PCE of $86 \%$, compared to $72 \%$ for the FX architecture. The enhanced performance at low input power (i.e., input power less than $-30 \mathrm{dBm}$ ) is exclusive for the DM architecture. For example, at $-35 \mathrm{dBm}$ input power, the PCE of the proposed DM rectifier is $38 \%$ which is more than two times the efficiency of the FX architecture. The superb performance at both the low and the high input power is reflected in the extended DR of the proposed rectifier. Note that, the DR is defined as the range where the performance of the rectifier exceeds $80 \%$ of the peak PCE. The DR is influenced by both the low and the high power performance of the rectifier and is calculated by:

$$
D R(d B)=P_{\text {max }}(d B m)-P_{\min }(d B m)
$$

where $P_{\max }$ and $P_{\text {min }}$ are the range where the PCE $\geq 0.8 \times$ peak PCE. Fig. 9(b) compares the peak PCE and the DR of the DM and the FX architectures for different loads. In general, the proposed design consistently offers improved performance compared to the FX architecture, with up to $19 \%$ enhancement in the peak PCE and 53\% in the DR.

Fig. 10 shows the measured output de versus the input power. The proposed rectifier offers a boosted output voltage across the full range of the input power. This improvement in the output voltage is reflected in the sensitivity of the rectifier defined as the input power required to generate a $1-\mathrm{V}$ output voltage at the load. The sensitivity of the proposed DM rectifier is $-19.2 \mathrm{dBm}$ which is more than $13 \mathrm{~dB}$ better than the FX

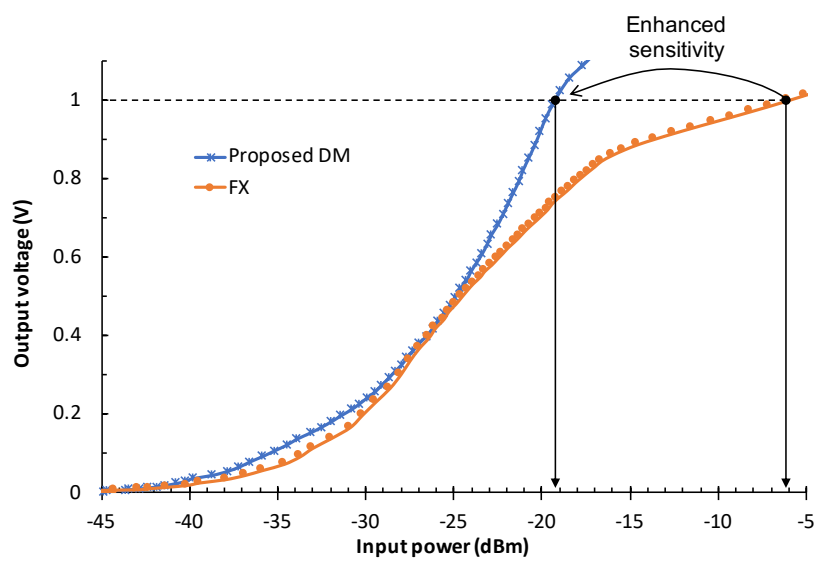

Fig. 10. Measured output voltage versus the input power with a $100 \mathrm{k} \Omega$ load.

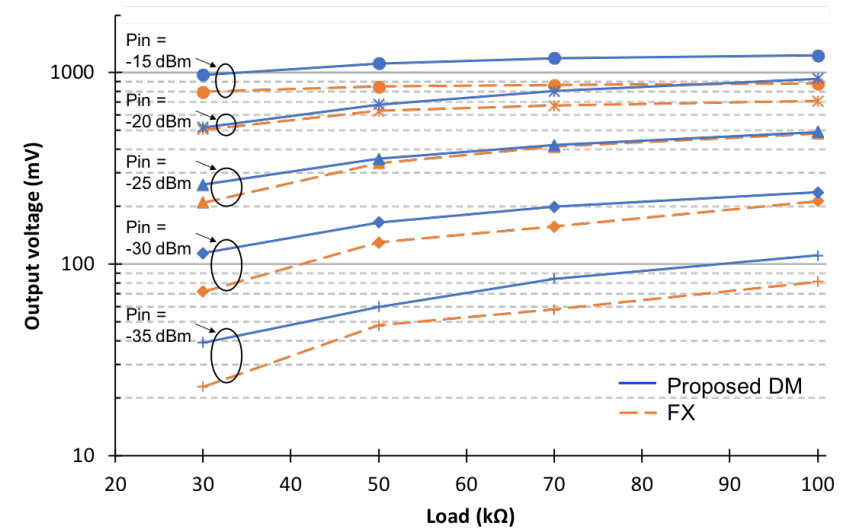

Fig. 11. Measured output voltage versus for different loads and input power. 
TABLE II

PERFORMANCE COMPARISON

\begin{tabular}{|c|c|c|c|c|c|c|c|c|c|}
\hline Architecture & $\begin{array}{l}\text { Load } \\
(\mathrm{k} \Omega)\end{array}$ & $\begin{array}{l}\text { Frequency } \\
\text { (MHz) }\end{array}$ & $\begin{array}{l}\text { Technology } \\
\quad(\mathrm{nm})\end{array}$ & $\begin{array}{l}\text { Number of } \\
\text { stages }\end{array}$ & $\begin{array}{c}\text { Area } \\
\left(\times 10^{3} \mu m^{2}\right)\end{array}$ & $\begin{array}{l}\text { Sensitivity }^{\mathrm{a}} \\
\quad(\mathrm{dBm})\end{array}$ & $\begin{array}{c}\operatorname{PCE}^{\text {b at }}-35 \mathrm{dBm} \\
(\%)\end{array}$ & $\begin{array}{c}\text { Peak } \\
\text { PCE } \\
(\%) \\
\end{array}$ & $\begin{array}{c}\text { Dynamic } \\
\text { Range }^{\mathrm{c}} \\
\text { (dB) }\end{array}$ \\
\hline Proposed design & 100 & 433 & 65 & 1 & 6.48 & -19.2 & 38 & 86 & 10.1 \\
\hline Self-biased [35] & 100 & 433 & 180 & 1 & 17 & -17 & $<3$ & 52 & 7.9 \\
\hline Diodes, double sided [40] & 100 & 433 & 180 & 1 & 8.8 & -19.2 & $<18$ & 86 & 6.7 \\
\hline Threshold compensated [48] & 100 & 433 & 180 & 4 & 150 & -13.5 & $<7$ & 34 & 8.2 \\
\hline $\begin{array}{l}\text { Dynamic Threshold Reduction } \\
\text { using PMOS [49] }\end{array}$ & 10 & 402 & 180 & 1 & 24 & $>-1$ & $<1$ & 86 & 4.2 \\
\hline $\begin{array}{l}\text { Adaptive Threshold Voltage } \\
\text { Compensation [46] }\end{array}$ & 30 & 402 & 180 & 3 & 1440 & -8.8 & N.A. & 31.9 & 10 \\
\hline Self-biased [50] & 50 & 850 & 180 & 1 & 17 & -N.A. & $<2$ & 40 & 6.48 \\
\hline Adaptive Vth compensated [51] & 130 & 915 & 130 & 12 & 250 & -17 & N.A. & 33 & 7.5 \\
\hline
\end{tabular}

a. Input RF power for $1-\mathrm{V}$ output voltage; ${ }^{\text {b. }}$ Power Conversion Efficiency (PCE); ${ }^{\text {c }}$ input power range of PCE $\geq 0.8 \times$ peak PCE; ${ }^{\text {d. }}$ measurement results are reproduced in this work; ${ }^{\mathrm{e} .}$ simulation results.

architecture. Fig. 11 shows the measured output voltage for different loads (ranging from $30 \mathrm{k} \Omega$ to $100 \mathrm{k} \Omega$ ) and input power levels (ranging from $-35 \mathrm{dBm}$ to $-15 \mathrm{dBm}$ ). In general, the proposed DM rectifier maintains the enhanced performance for
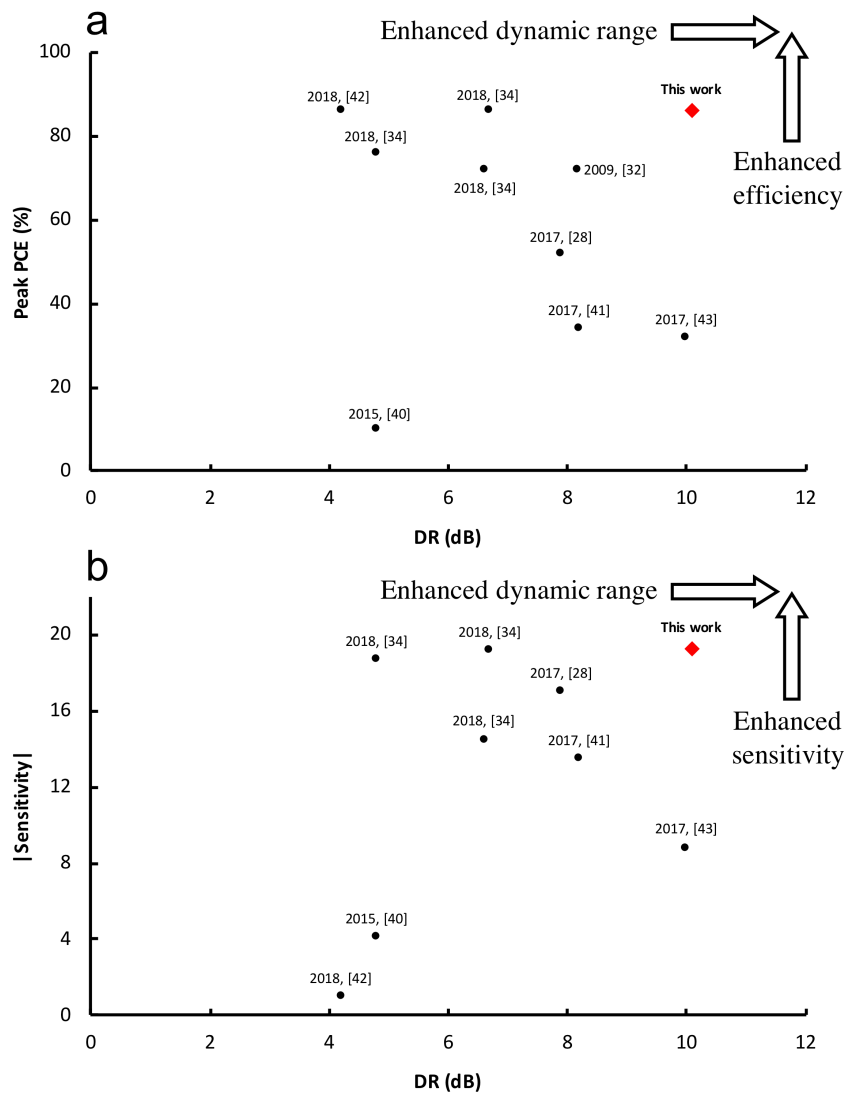

Fig. 12. (a) Peak PCE and (b) sensitivity versus DR for different architectures operating at the UHF band. the large range of the input power and the resistive loads.

Table II compares the performance of the proposed DM rectifier with the state-of-the-art rectifiers designed in CMOS technology and operating at similar frequency range. The proposed design offers the best sensitivity, peak PCE and DR. Moreover, the proposed design offers the best low-power performance with a PCE of $57 \%$ at $-30 \mathrm{dBm}$. The double-sided architecture [40] offers a similar peak PCE and sensitivity, yet, the proposed design offers $3.4 \mathrm{~dB}$ wider $\mathrm{DR}$ and $37 \%$ higher PCE at $-30 \mathrm{dBm}$ input power due to the superb low power performance of the proposed architecture. The adaptive threshold voltage compensation [46] offers similar DR to the proposed design, yet, the proposed design offers more than double the peak PCE. Fig. 12 compares the performances of many recent architectures including the peak PCE and the sensitivty versus the DR operating at similler frequency range (i.e., 400 to $500 \mathrm{MHz}$ ). In general, the proposed DM rectifier offers consistently the highest PCE and the best sensitivity for a wide range of RF power levels.

\section{CONCLUSION}

The proposed dual-mode nested feedback circuit enhances the low- and high- power performance of the fully crosscoupled architecture by dynamically changing the dc operating points of the rectifying transistors. At low input power, it applies a $V_{S S}$ dc voltage at the gates of the PMOS rectifying transistors to enhance their conductivity. On the other hand, the reverse leakage current at high input power is reduced by applying a $V_{D D}$ dc voltage at the gates of the PMOS rectifying transistors. The proposed DM rectifier is implemented in $65 \mathrm{~nm}$ CMOS technology using low- and high- threshold transistors. Nevertheless, this topology is scalable to other CMOS 
technologies that support low-threshold transistors. The proposed dual-mode rectifier offers a high peak power conversion efficiency of $86 \%$ with exceptionally low-power performance exceeding $10 \%$ for input power greater than -40 $\mathrm{dBm}$. Such a a design enables ampient wireless powering and energy harvesting using standard CMOS technology.

\section{ACKNOWLEDGMENT}

The authors thank Dr. M. Ouda and Mr. Nguyen Q. Thang for their generous support and valuable feedback.

\section{References}

[1] B. Haug, "Wireless Sensor Nodes Can Be Powered by Temperature Gradients; No Batteries Needed: Harvesting Energy from Thermoelectric Generators," IEEE Power Electron. Mag., vol. 4, no. 4, pp. 24-32, Dec. 2017.

[2] Q. Ju and Y. Zhang, "Predictive power management for Internet of battery-less things," IEEE Trans. Power Electron., vol. 33, no. 1, pp. 299312, Jan. 2018.

[3] S. Selvan, M. Zaman, R. Gobbi, and H. Y. Wong, "Recent advances in the design and development of radio frequency-based energy harvester for powering wireless sensors: a review," Journal of Electromagnetic Waves and Applications, vol. 32, no. 16, pp. 1-25, 2018.

[4] M. Zaman, H. Y. Wong, M. Islam, and N. Amin, "An integrated hybrid energy harvester for autonomous wireless sensor network nodes," International Journal of Photoenergy, vol. 2014, 2014.

[5] P. Saffari, A. Basaligheh, V. J. Sieben, and K. Moez, "An RF-powered wireless temperature sensor for harsh environment monitoring with nonintermittent operation," IEEE Trans. Circuits Sys. I: Reg. Papers, vol. 65, no. 5, pp. 1529-1542, May 2018.

[6] MarketsAndMarkets. "Wireless Sensor Network Market by Offering (Hardware, Software, Services), Sensor Type, Connectivity Type, Enduser Industry (Building Automation, Wearable Devices, Healthcare, Automotive \& Transportation, Industrial), and Region - Global Forecast to 2023." https://www.marketsandmarkets.com/Market-Reports/wirelesssensor-networks-market-445.html

[7] C. R. Valenta and G. D. Durgin, "Harvesting wireless power: Survey of energy-harvester conversion efficiency in far-field, wireless power transfer systems," IEEE Microw. Mag., vol. 15, no. 4, pp. 108-120, 2014.

[8] M. H. Ouda, M. Arsalan, L. Marnat, A. Shamim, and K. N. Salama, "5.2GHz RF Power Harvester in 0.18- $\mu \mathrm{m}$ CMOS for Implantable Intraocular Pressure Monitoring," IEEE Trans. Microw. Theory Techn., vol. 61, no. 5, pp. 2177-2184, May 2013.

[9] Y.-J. Kim, H. S. Bhamra, J. Joseph, and P. P. Irazoqui, "An ultra-lowpower RF energy-harvesting transceiver for multiple-node sensor application," IEEE Trans. Circuits Sys. II: Exp. Briefs, vol. 62, no. 11, pp. 1028-1032, Nov. 2015.

[10] O. Elsayed, M. Abouzied, V. Vaidya, K. Ravichandran, and E. SánchezSinencio, "An Ultralow-Power RF Wireless Receiver With RF Blocker Energy Recycling for IoT Applications," IEEE Trans. Microw. Theory Techn., vol. 66, no. 11, pp. 4927-4942, Nov. 2018.

[11] T. Ngo, A. Huang, and Y. X. Guo, "Analysis and Design of a Reconfigurable Rectifier Circuit for Wireless Power Transfer," IEEE Trans. Ind. Electron., vol. 66, no. 9, pp. 7089-7098, Sept. 2019.

[12] S.-Y. Kim et al., "A-20 to $30 \mathrm{dBm}$ Input Power Range Wireless Power System with a MPPT-based Reconfigurable $48 \%$ Efficient RF Energy Harvester and $82 \%$ Efficient A4WP Wireless Power Receiver with Open Loop Delay Compensation," IEEE Trans. Power Electron., vol. 34, no. 7, pp. 6803-6817, July 2019.

[13] L. Marnat, M. Ouda, M. Arsalan, K. Salama, and A. Shamim, "On-chip implantable antennas for wireless power and data transfer in a glaucomamonitoring SoC," IEEE Antennas Wireless Propag. Lett., vol. 11, pp. 1671-1674, 2012.

[14] P. Burasa, N. G. Constantin, and K. Wu, "High-efficiency wideband rectifier for single-chip batteryless active millimeter-wave identification (MMID) tag in 65-nm bulk CMOS technology," IEEE Trans. Microw. Theory Techn., vol. 62, no. 4, pp. 1005-1011, April 2014.

[15] B. Clerckx, R. Zhang, R. Schober, D. W. K. Ng, D. I. Kim, and H. V. Poor, "Fundamentals of wireless information and power transfer: From
RF energy harvester models to signal and system designs," IEEE J. Sel. Areas Commun., vol. 37, no. 1, pp. 4-33, Jan. 2019.

[16] M. Arsalan, M. H. Ouda, L. Marnat, T. J. Ahmad, A. Shamim, and K. N. Salama, "A 5.2 GHz, 0.5 mW RF powered wireless sensor with dual onchip antennas for implantable intraocular pressure monitoring," in IEEE MTT-S Int. Microw. Symp. Dig. (MTT), Jun. 2013, pp. 1-4.

[17] J. Koomey, S. Berard, M. Sanchez, and H. Wong, "Implications of historical trends in the electrical efficiency of computing," IEEE Ann. Hist. Comput., vol. 33, no. 3, pp. 46-54, March 2011.

[18] S. Hemour et al., "Towards low-power high-efficiency RF and microwave energy harvesting," IEEE Trans. Microw. Theory Techn., vol. 62, no. 4, pp. 965-976, April 2014.

[19] A. Bakytbekov, T. Q. Nguyen, C. Huynh, K. N. Salama, and A. Shamim, "Fully printed 3D cube-shaped multiband fractal rectenna for ambient RF energy harvesting," Nano Energy, vol. 53, pp. 587-595, Nov. 2018.

[20] M. Stoopman, K. Philips, and W. A. Serdijn, "An RF-powered DLLbased 2.4-GHz transmitter for autonomous wireless sensor nodes," IEEE Trans. Microw. Theory Techn., vol. 65, no. 7, pp. 2399-2408, July 2017.

[21] K. R. Sadagopan, J. Kang, Y. Ramadass, and A. Natarajan, "A cm-Scale 2.4-GHz Wireless Energy Harvester With NanoWatt Boost Converter and Antenna-Rectifier Resonance for WiFi Powering of Sensor Nodes," IEEE J. Solid-State Circuits, vol. 53, no. 12, pp. 3396-3406, Dec. 2018.

[22] D. Bao, Z. Zou, M. B. Nejad, Y. Qin, and L.-R. Zheng, "A Wirelessly Powered UWB RFID Sensor Tag With Time-Domain Analog-toInformation Interface," IEEE J. Solid-State Circuits, vol. 53, no. 8, pp. 2227-2239, Aug. 2018.

[23] S. Fan, R. Wei, L. Zhao, X. Yang, L. Geng, and P. X.-L. Feng, "An Ultralow Quiescent Current Power Management System With Maximum Power Point Tracking (MPPT) for Batteryless Wireless Sensor Applications," IEEE Trans. Power Electron., vol. 33, no. 9, pp. 73267337, Sept. 2018.

[24] M. Chen et al., "A Self-Powered 3.26- $\mathrm{W}$ 70-meter Wireless Temperature Sensor Node for Power Grid Monitoring," IEEE Trans. Ind. Electron., vol. 65, no. 11, pp. 8956-8965, Nov. 2018.

[25] S. A. Mirbozorgi, P. Yeon, and M. Ghovanloo, "Robust wireless power transmission to $\mathrm{mm}$-sized free-floating distributed implants," IEEE Trans. Biomed. Circuits Sys., vol. 11, no. 3, pp. 692-702, June 2017.

[26] B. Lee, M. Kiani, and M. Ghovanloo, "A smart wirelessly powered homecage for long-term high-throughput behavioral experiments," IEEE Sensors J., vol. 15, no. 9, pp. 4905-4916, Sept. 2015.

[27] A. M. Baranov, S. Akbari, D. Spirjakin, A. Bragar, and A. Karelin, "Feasibility of RF energy harvesting for wireless gas sensor nodes," Sensors and Actuators A: Physical, vol. 275, pp. 37-43, June 2018.

[28] M. Arsalan, M. H. Ouda, L. Marnat, A. Shamim, and K. N. Salama, "Implantable intraocular pressure monitoring systems: Design considerations," in Proc. IEEE MTT-S Int. Microw. Workshop Ser., RF Wireless Technol. Biomed. Healthcare Appl. (IMWS-BIO), Dec. 2013, pp. $1-3$.

[29] Y. Zhang, L. T. Yang, and J. Chen, RFID and sensor networks: architectures, protocols, security, and integrations. CRC Press, 2009.

[30] J. Zhu, L. Wu, X. Zhang, C. Jia, and C. Zhang, "A low-power $433 \mathrm{MHz}$ transmitter for battery-less Tire Pressure Monitoring System," in Proc. IEEE 9th Int. Conf. ASICON, Xiamen, 2011, Oct. pp. 184-187.

[31] A. Abdou et al., "A matched Bow-tie antenna at $433 \mathrm{MHz}$ for use in underwater wireless sensor networks," in J. Phys.: Conf. Ser., vol. 450, no. 1,2013 , Art. no. 012048

[32] A. Almansouri, M. Ouda, and K. N. Salama, "A highly sensitive RF-toDC power converter with an extended dynamic range," in Proc. IEEE 60th Int. Midwest Symp. Circuits Sys. (MWSCAS), Boston, MA, USA, Aug. 2017, pp. 436-439.

[33] J. F. Dickson, "On-chip high-voltage generation in MNOS integrated circuits using an improved voltage multiplier technique," IEEE J. SolidState Circuits, vol. 11, no. 3, pp. 374-378, June 1976.

[34] A. S. Sedra and K. C. Smith, "Microelectronic Circuits," 2015.

[35] M. H. Ouda, W. Khalil, and K. N. Salama, "Self-biased differential rectifier with enhanced dynamic range for wireless powering," IEEE Trans. Circuits Sys. II: Exp. Briefs, vol. 64, no. 5, pp. 515-519, May 2017.

[36] C. H. P. Lorenz et al., "Breaking the efficiency barrier for ambient microwave power harvesting with heterojunction backward tunnel diodes," IEEE Trans. Microw. Theory Techn., vol. 63, no. 12, pp. 45444555, Dec. 2015.

[37] K. Kotani, A. Sasaki, and T. Ito, "High-efficiency differential-drive CMOS rectifier for UHF RFIDs," IEEE J. Solid-State Circuits, vol. 44, no. 11, pp. 3011-3018, Nov. 2009. 
[38] M. H. Ouda, W. Khalil, and K. N. Salama, "Wide-range adaptive RF-toDC power converter for UHF RFIDs," IEEE Microw. Wireless Compon. Lett., vol. 26, no. 8, pp. 634-636, Aug. 2016.

[39] X. Yu, K. Moez, I.-C. Wey, M. Sawan, and J. Chen, "A fully integrated multistage cross-coupled voltage multiplier with no reversion power loss in a standard CMOS process," IEEE Trans. Circuits Sys. II: Exp. Briefs, vol. 64 , no. 7, pp. 737-741, July 2017.

[40] A. S. Almansouri, M. H. Ouda, and K. N. Salama, "A CMOS RF-to-DC Power Converter With $86 \%$ Efficiency and-19.2-dBm Sensitivity," IEEE Trans. Microw. Theory Techn., vol. 66, no. 5, pp. 2409-2415, May 2018.

[41] U. Guler and M. Ghovanloo, "Power Management in Wireless PowerSipping Devices: A Survey," IEEE Circuits Sys. Mag., vol. 17, no. 4, pp. 64-82, Fourthquarter 2017.

[42] N. H. Weste and D. Harris, CMOS VLSI design: a circuits and systems perspective. Pearson Education India, 2015.

[43] Z. Liu, Y.-P. Hsu, B. Fahs, and M. M. Hella, "An RF-DC Converter IC With On-Chip Adaptive Impedance Matching and 307- $\mu \mathrm{W}$ Peak Output Power for Health Monitoring Applications," IEEE Trans. Trans. Very Large Scale Integr. (VLSI) Syst., vol. 26, no. 8, pp. 1565-1574, Aug. 2018.

[44] M. Stoopman, S. Keyrouz, H. J. Visser, K. Philips, and W. A. Serdijn, "Co-design of a CMOS rectifier and small loop antenna for highly sensitive RF energy harvesters," IEEE J. Solid-State Circuits, vol. 49, no. 3, pp. 622-634, March 2014.

[45] S. Mandal and R. Sarpeshkar, "Low-power CMOS rectifier design for RFID applications," IEEE Trans. Circuits Sys. I: Reg. Papers, vol. 54, no. 6, pp. 1177-1188, June 2007.

[46] Y.-S. Luo and S.-I. Liu, "A Voltage Multiplier With Adaptive Threshold Voltage Compensation," IEEE J. Solid-State Circuits, vol. 52, no. 8, pp. 2208-2214, Aug. 2017

[47] S. S. Chouhan and K. Halonen, "A novel cascading scheme to improve the performance of voltage multiplier circuits," Analog Integ. Circuits Sig. Process., vol. 84, no. 3, pp. 373-381, Sept. 2015.

[48] K. Gharehbaghi, Ö. Zorlu, F. Koçer, and H. Külah, "Threshold Compensated UHF Rectifier With Local Self-Calibrator," IEEE Microw. Wireless Compon. Lett., vol. 27, no. 6, pp. 575-577, June 2017.

[49] M. M. Mohamed et al., "High-Efficiency CMOS RF-to-DC Rectifier Based on Dynamic Threshold Reduction Technique for Wireless Charging Applications," IEEE Access, vol. 6, pp. 46826-46832, 2018.

[50] M. Ouda, "Wide-range highly-efficient wireless power receivers for implantable biomedical sensors," Ph.D. dissertation, Dept. Comput., Electr. Math. Sci. Eng. (CEMSE) Division, King Abdullah Univ. Sci. Technol., Thuwal, Saudi Arabia, 2016.

[51] Z. Hameed and K. Moez, "A $3.2 \mathrm{~V}-15 \mathrm{dBm}$ adaptive threshold-voltage compensated RF energy harvester in $130 \mathrm{~nm}$ CMOS," IEEE Trans. Circuits Sys. I: Reg. Papers, vol. 62, no. 4, pp. 948-956, April 2015.

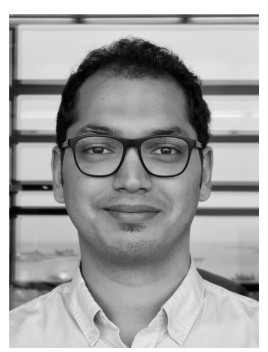

Abdullah S. Almansouri (GS'16) received the B.Sc. degree (with honors) from Royal Melbourne Institute of Technology University (RMIT), Melbourne, VIC, Australia, in 2013, and the M.S. degree from King Abdullah University of Science and Technology (KAUST), Saudi Arabia, in 2016. He is currently working toward the Ph.D.

degree at the same university.

His research interests revolve around wireless sensors, including wireless powering using CMOS technology, energy harvesting, and low-power wearable sensors. Mr. Almansouri was recognized by the Golden Key International Honour Society as the top $15 \%$ of students worldwide in the field of Electronics and Communication in 2013; ranked first in Saudi Arabia for the 10th Annual International Microelectronics Olympiad of Armenia in 2015; and attended the 2019 Lindau Nobel Laureate meeting.

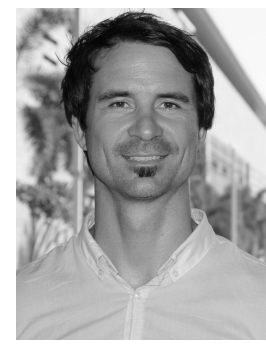

Jürgen Kosel (SM'14) received the Dipl.Ing. (M.Sc.) and D.Sc. degrees in electrical engineering from the Vienna University of Technology, Austria, in 2002 and 2006, respectively. $\mathrm{He}$ is Associate Professor of Electrical Engineering at KAUST since 2015, where he is with the Computer, Electrical and Mathematical Sciences and Engineering Division. He joined KAUST as Assistant Professor in 2009. He is the Head of the Sensing, Magnetism and Microsystems Research Group. His research interests are in the fields of micro- and nano devices with a focus on magnetic transducers.

He was a Post-Doctoral Fellow with the Biomedical Engineering Research Group, Stellenbosch University, South Africa, from 2007 to 2009, where he conducted research in the fields of Bio-MEMS for HIV detection, magnetic microwires for stress measurements in body tissues, and kinematics and mechanics of the knee joint. In 2006 and 2007, he was with the automotive industry as a Project Manager at Magna Powertrain in Austria. During his doctoral work, he developed a multifunctional bending sensor based on magnetostrictive materials for biomedical and automotive applications. In addition, he conducted research in electrical concentration and detection of microorganisms, magnetically operated microgrippers, as well as test systems for rotational microdevices.

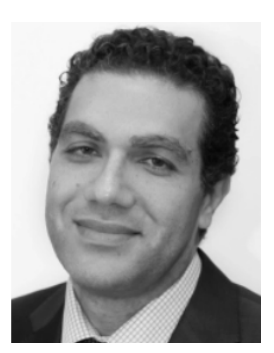

Khaled N. Salama (S'97-M'05-SM'10) received the B.S. degree from the Department Electronics and Communications, Cairo University, Cairo, Egypt, in 1997, and the M.S. and Ph.D. degrees from the Department of Electrical Engineering, Stanford University, Stanford, CA, USA, in 2000 and 2005, respectively. He was an Assistant Professor at Rensselaer Polytechnic Institute, NY, USA, between 2005 and 2009. He joined King Abdullah University of Science and Technology (KAUST) in January 2009, where he is now a professor, and was the founding Program Chair until August 2011. He is the director of the sensors initiative a consortium of 9 universities (KAUST, MIT, UCLA, GATECH, Brown University, Georgia Tech, TU Delft, Swansea University, the University of Regensburg and the Australian Institute of Marine Science (AIMS)).

His work on CMOS sensors for molecular detection has been funded by the National Institutes of Health (NIH) and the Defense Advanced Research Projects Agency (DARPA), awarded the Stanford-Berkeley Innovators Challenge Award in biological sciences and was acquired by Illumina Inc. He is the author of 250 papers and 14 US patents on low-power mixedsignal circuits for intelligent fully integrated sensors and neuromorphic circuits using memristor devices. 